\title{
A stabilized finite element method for incompressible viscous flows using a finite increment calculus formulation
}

\author{
Eugenio Oñate * \\ International Centre for Numerical Methods in Engineering, Universidad Politécnica de Cataluña, Gran Capitán s/n, 08034 Barcelona, \\ Spain \\ Received 1 September 1998
}

\begin{abstract}
A stabilized finite element formulation for incompressible viscous flows is derived. The starting point are the modified NavierStokes equations incorporating naturally the necessary stabilization terms via a finite increment calculus (FIC) procedure. Application of the standard finite element Galerkin method to the modified differential equations leads to a stabilized discrete system of equations overcoming the numerical instabilities emanating from the advective terms and those due to the lack of compatibility between approximate velocity and pressure fields. The FIC method also provides a natural explanation for the stabilization terms appearing in all equations for both the Navier-Stokes and the simpler Stokes equations. Transient solution schemes with enhanced stabilization properties are also proposed. Finally a procedure for computing the stabilization parameters is presented. (C) 2000 Elsevier Science S.A. All rights reserved.
\end{abstract}

\section{Introduction}

Finite element solution of the incompressible Navier-Stokes equations with the classical Galerkin method may suffer from numerical instabilities from two main sources. The first is due to the advectivediffusive character of the equations which induces oscillations for high values of the velocity. The second source has to do with the mixed character of the equations which limits the choice of finite element interpolations for the velocity and pressure fields [1].

Solutions of these two problems have been extensively sought in the last years. Compatible velocitypressure interpolations satisfying the inf-sup condition emanating from the second problem above mentioned have been used [1,2]. In addition, the advective operator has been modified to include some "upwinding" effects [3-8]. Recent procedures based on Galerkin Least Square techniques [9,7] allow equal order interpolation for velocity and pressure by introducing a Laplacian of pressure term in the mass balance equation, while preserving the upwinding stabilization of the momentum equations. Similar effects can be obtained using Characteristic Galerkin methods [10,11], Variational Multiscale models [12,13] and analogous Residual-Free Bubbles techniques [14-16]. Most of these methods lack enough stability in the presence of sharp layers transversal to the velocity. This defficiency is usually corrected by adding new "shock capturing" stabilization terms to the already stabilized equations [17-20]. The computation of the stabilization parameters in all these methods is mostly based in "ad hoc" generalizations of the 1D linear

\footnotetext{
${ }^{*}$ Tel.: +34-93-205-7016; fax: +34-93-401-6517.

E-mail address: onate@cimne.upc.es (E. Oñate).
} 
advective-diffusive problem. Despite several recent attempts there still lacks a general methodology for evaluating the stabilization parameters for fluid flow problems in an objective and accurate manner.

This paper presents a different point view for deriving stabilized finite element methods for incompressible flow problems. The starting point are the stabilized form of the governing differential equations derived via a finite increment calculus (FIC) procedure. This technique presented in [21-25] is based on writting the momentum and mass balance equations over a domain of finite size and retaining higher order terms. These terms incorporate the ingredients for the necessary stabilization of any numerical solution already at the differential equations level. Application of the standard Galerkin formulation to the consistently modified differential equations leads to a stabilized system of discretized equations which overcomes the two problems above mentioned (i.e., the advective type instability and that due to lack of compatibility between the velocity and pressure fields). In addition, the modified differential equations can be used to derive a numerical scheme for iteratively computing the stabilization parameters in a sort of model adaptivity procedure [22-25].

The paper is structured as follows. In Section 2 the derivation of the stabilized modified differential equations for incompressible Navier-Stokes flows using the FIC method is presented. Details of the stabilized finite element formulation are then given. The case of simpler Stokes flows is considered next. Indeed the stabilized formulation obtained in this case should also be useful for solving the analogous incompressible elasticity problem. A three steps time marching solution scheme with enhanced stabilization properties is derived starting from the stabilization differential equations for the transient case. In the last part of the paper a procedure for computing the stabilization parameters is proposed.

2. Stabilized governing equations for incompressible flows

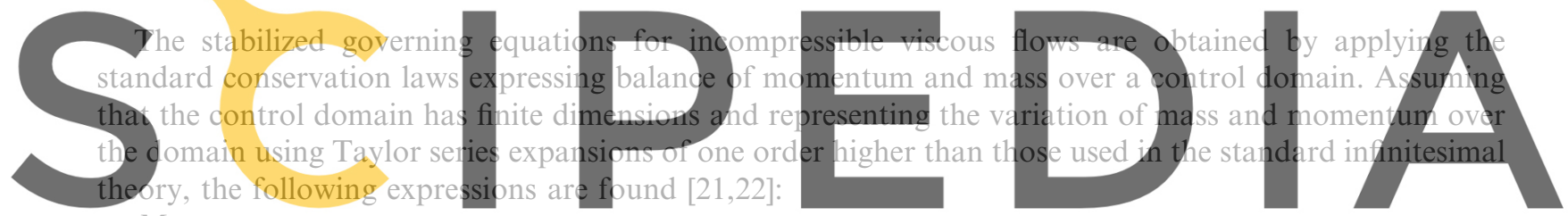

Momentum

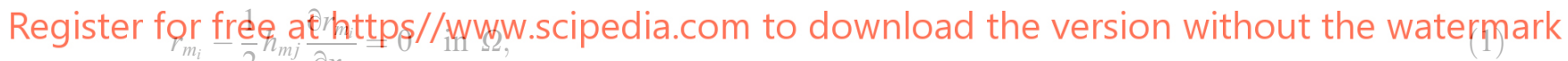
$2^{n j} \frac{\partial x_{j}}{}$

Mass balance

$$
r_{d}-\underline{\frac{1}{2} h_{d j} \frac{\partial r_{d}}{\partial x_{j}}}=0 \text { in } \Omega
$$

where for the steady state case

$$
\begin{aligned}
& r_{m_{i}}=\rho \frac{\partial\left(u_{i} u_{j}\right)}{\partial x_{j}}+\frac{\partial p}{\partial x_{i}}-\frac{\partial \tau_{i j}}{\partial x_{j}}-b_{i}, \\
& r_{d}=\frac{\partial u_{i}}{\partial x_{i}}
\end{aligned}
$$

with $i, j=1,2$ for a two dimensional flow.

In Eq. (3) $\rho$ is the fluid density (here assumed to be constant), $u_{i}$ is the velocity component in the $i$ th direction, $p$ the pressure, $b_{i}$ the body forces and $\tau_{i j}$ the viscous stress components related to the velocity gradients through the fluid viscosity $\mu$ by

$$
\tau_{i j}=2 \mu\left(\varepsilon_{i j}-\frac{1}{3} \frac{\partial u_{k}}{\partial x_{k}} \delta_{i j}\right)
$$


with

$$
\varepsilon_{i j}=\frac{1}{2}\left(\frac{\partial u_{i}}{\partial x_{j}}+\frac{\partial u_{j}}{\partial x_{i}}\right) .
$$

Einstein summation convention for repeated indexes in products and derivatives is used, i.e., $h_{d j}\left(\partial r_{d} / \partial x_{j}\right)=\sum_{j} h_{d j}\left(\partial r_{d} / \partial x_{j}\right)$.

Eqs. (1) and (2) are the stabilized forms of the governing differential equations for an incompressible flow. The terms underlined in (1) and (2) introduce naturally the necessary stabilization at the discretization level. The so called characteristic length vectors $\mathbf{h}_{m}$ and $\mathbf{h}_{d}$ are defined as (for 2D problems)

$$
\mathbf{h}_{m}=\left\{\begin{array}{l}
h_{m_{1}} \\
h_{m_{2}}
\end{array}\right\}, \quad \mathbf{h}_{d}=\left\{\begin{array}{l}
h_{d_{1}} \\
h_{d_{2}}
\end{array}\right\},
$$

where $h_{m_{1}}$ and $h_{m_{2}}$ are the dimensions of the finite control domain where balance of momentum is enforced. Similarly $h_{d_{1}}$ and $h_{d_{2}}$ represent the dimensions of the domain where mass conservation is expressed. The components of vectors $\mathbf{h}_{m}$ and $\mathbf{h}_{d}$ introduce the necessary stabilization along the streamline and transverse directions to the flow in the discrete problem.

The method to derive the modified differential Eqs. (1) and (2) incorporating the stabilization terms was termed in [22] FIC as a reference to the standard infinitesimal calculus techniques where the size of the domain where balance of mass and momentum is enforced is assumed to be negligible. Note that for $\mathbf{h}_{m}=\mathbf{h}_{d} \rightarrow 0$ the standard infinitesimal form of the momentum and mass balance equations is recovered $[21,22]$

Eqs. (1) and (2) are complemented by the following boundary conditions [21,22].

Balance of momentum at the boundary $\Gamma_{t}$

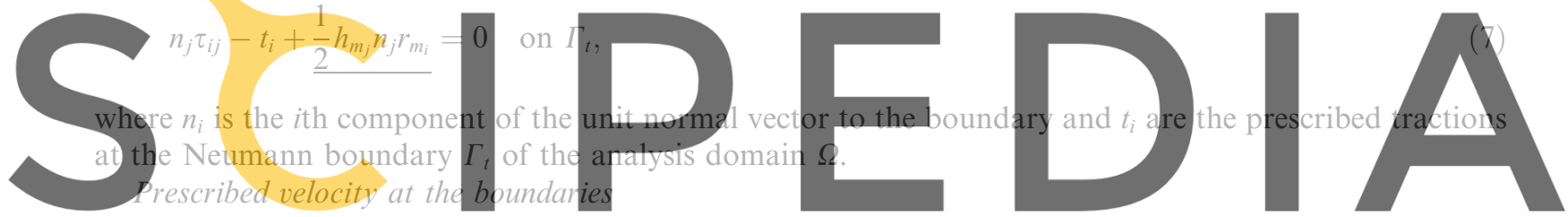

$u_{t}=u_{t}^{p}$ on $\Gamma_{u_{t}}$,
for free at https//www.scipedia.com to download the version without the watermark

Register for free at https//www.scipedia.com to download the version without the watermark

$$
u_{n}-\frac{1}{2} h_{d_{i}} n_{i} r_{d}=u_{n}^{p} \quad \text { on } \Gamma_{u_{n}} .
$$

In Eq. (8) $u_{t}$ and $u_{t}^{p}$ denote the tangential velocity to the boundary and its prescribed value, respectively.

Eq. (9) expresses the balance of mass on an arbitrary domain next to the boundary. In Eq. (9) $u_{n}$ and $u_{n}^{p}$ denote the velocity normal to the boundary and its prescribed value, respectively. The value of $u_{n}^{p}$ is zero on solid walls and stationary free surfaces.

Also in Eqs. (8) and (9) $\Gamma_{u_{t}}$ and $\Gamma_{u_{n}}$ are the parts of the boundary $\Gamma$ of $\Omega$ where the tangential and normal velocities are prescribed, respectively. The Dirichlet boundary is defined as $\Gamma_{u}=\Gamma_{u_{t}} \cup \Gamma_{u_{n}}$.

The underlined terms in Eqs. (7) and (8) introduce the necessary stabilization at the boundaries in a form consistent with that of Eqs. (1) and (2). These terms are obtained by invoking balance of momentum and mass at a domain of finite size next to the boundary. Details of the derivation of Eqs. (1)-(8), can be found in $[21,22]$ whereas the derivation of Eq. (9) is shown in Appendix A.

\subsection{Alternative form of stabilized governing equations}

Let us express the components of the characteristic vector $\mathbf{h}_{d}$ for the mass balance equation as

$$
h_{d_{i}}=-2 \rho \tau_{d_{i}} u_{i},
$$

where the $\tau_{d_{i}}$ parameters are termed "intrinsic times" per unit mass. The negative sign in Eq. (10) is necessary to introduce a positive stabilization in the mass balance equation at the discrete level as it will be shown later. 
From simple differentiation rules we can write

$$
u_{i} \frac{\partial}{\partial x_{i}}\left(\frac{\partial u_{j}}{\partial x_{j}}\right)=\frac{\partial}{\partial x_{i}}\left(u_{i} \frac{\partial u_{j}}{\partial x_{j}}\right)-\left(\frac{\partial u_{k}}{\partial x_{k}}\right)^{2} .
$$

Substituting Eq. (10) into Eq. (2) and making use of Eqs. (11), (1) and (3) we can rewrite the mass balance equation (neglecting higher order terms) as

$$
r_{d}-\tau_{d i} \frac{\partial \hat{r}_{m_{i}}}{\partial x_{i}}=0
$$

where

$$
\hat{r}_{m_{i}}=\rho u_{j} \frac{\partial u_{i}}{\partial x_{j}}+\frac{\partial p}{\partial x_{i}}-\frac{\partial \tau_{i j}}{\partial x_{j}}-b_{i} .
$$

Following a similar process, Eq. (9) expressing balance of mass at the boundary can be rewritten using Eqs. (1) and (10) as

$$
u_{n}-\tau_{d_{i}} n_{i} \hat{r}_{m_{i}}=u u_{n}^{p} \text { on } \Gamma_{u_{n}}
$$

We summarize next for the sake of clarity the set of governing equations to be solved.

\section{Momentum}

$$
r_{m_{i}}-\frac{1}{2} h_{m_{j}} \frac{\partial r_{m_{i}}}{\partial x_{j}}=0 \text { in } \Omega
$$
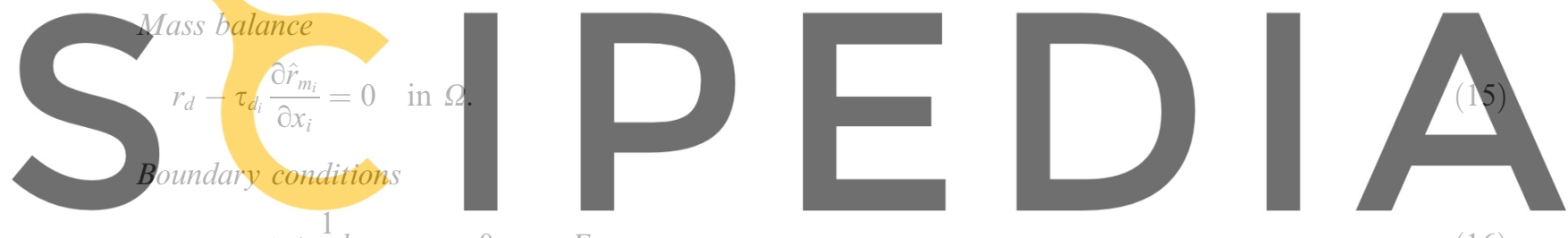

$n_{j} \sigma_{i j}-t_{i}+\frac{1}{2} h_{m_{j}} n_{j} r_{m_{i}}=0$ on $\Gamma_{t}$,

(16)

Register for free at $\mathrm{https//www.scipedia.com} \mathrm{to} \mathrm{download} \mathrm{the} \mathrm{version} \mathrm{without} \mathrm{the} \mathrm{watermark}$

$$
\begin{aligned}
& u_{t}-u_{t}^{p}=0 \quad \text { on } \Gamma_{u_{t}}, \\
& u_{n}-\tau_{d_{i}} n_{i} \hat{r}_{m_{i}}-u_{n}^{p}=0 \quad \text { on } \Gamma_{u_{n}},
\end{aligned}
$$

where $r_{m_{i}}$ and $\hat{r}_{m_{i}}$ are defined in Eqs. (3) and (12b), respectively.

A similar form of the modified differential equations for momentum and mass balance (Eqs. (14) and (15)) has been recently proposed by Ilinca et al. [26]. They express the exact solution as sum of the numerical approximation and a perturbation. The modified equations are derived by expanding the original differential equations for momentum and mass balance in Taylor series and eliminating the perturbation terms. However, the boundary conditions remain unchanged and thus the stabilizing terms in Eqs. (16) and (18) are omitted in [26]. This leads to the appearance of additional boundary integrals in the Galerkin formulation. These terms vanish naturally if the full stabilized expressions (14)-(18) emanating from the FIC method are used as shown in Section 3.

\section{Finite element formulation}

Let us now introduce a standard finite element interpolation of the velocity and pressure fields written as

$$
u_{i} \simeq \bar{u}_{i}=\sum_{j=1}^{n} N_{j}^{u}\left(\bar{u}_{i}\right)_{j}
$$




$$
p \simeq \bar{p}=\sum_{j=1}^{n} N_{j}^{p} \bar{p}_{j}
$$

where $N_{i}^{u}$ and $N_{i}^{p}$ are the shape functions interpolating the velocity $u_{i}$ and the pressure $p$ within each element and $(\bar{\cdot})_{j}$ denotes nodal values [1]. The numerical solution residuals are now defined as

$$
\begin{aligned}
& \bar{r}_{m_{i}}=r_{m_{i}}\left(\bar{u}_{i}, \bar{p}\right), \\
& \hat{\bar{r}}_{m_{i}}=\hat{r}_{m_{i}}\left(\bar{u}_{i}, \bar{p}\right), \\
& \bar{r}_{d}=r_{d}\left(\bar{u}_{i}\right) .
\end{aligned}
$$

Let us next apply the standard weighted residual method to the discretized stabilized governing equations (14)-(18). This gives

Momentum

$$
\int_{\Omega} v_{k}\left[\bar{r}_{m_{i}}-\frac{1}{2} h_{m_{j}} \frac{\partial \bar{r}_{m_{i}}}{\partial x_{j}}\right] \mathrm{d} \Omega+\int_{\Gamma_{t}} \hat{v}_{k}\left[n_{j} \bar{\sigma}_{i j}-t_{i}+\frac{1}{2} h_{m_{j}} n_{j} \bar{r}_{m_{i}}\right] \mathrm{d} \Gamma=0 .
$$

$$
\begin{aligned}
& \text { Mass balance } \\
& \qquad \int_{\Omega} q\left[\bar{r}_{d}-\tau_{d_{i}} \frac{\partial \hat{\bar{r}}_{m_{i}}}{\partial x_{i}}\right] \mathrm{d} \Omega+\int_{\Gamma_{u n}} \hat{q}\left[\bar{u}_{n}-\tau_{d_{i}} n_{i} \hat{\bar{r}}_{m_{i}}-u_{n}^{p}\right] \mathrm{d} \Gamma=0 .
\end{aligned}
$$

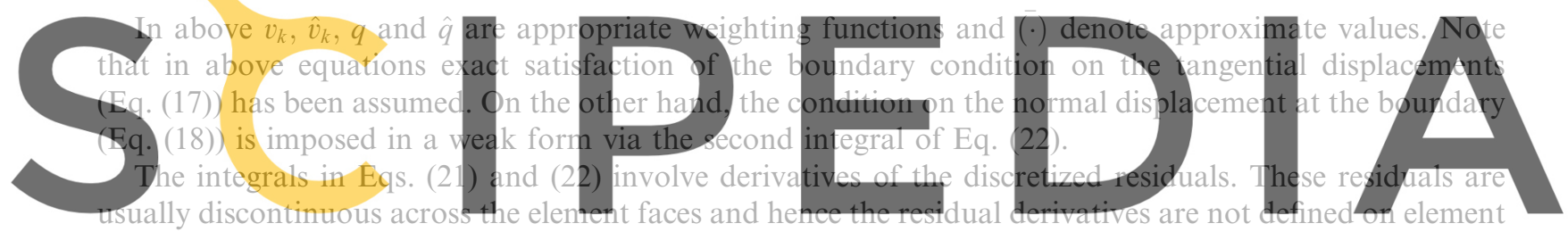

boundaries. This problem can be simply overcome by computing these derivatives in a distributional sense

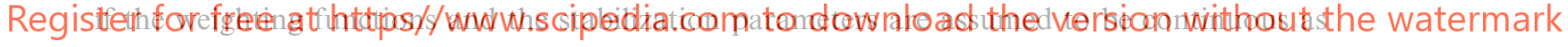

$$
\begin{aligned}
& \int_{\Omega} v_{k} h_{m_{j}} \frac{\partial \bar{r}_{m_{i}}}{\partial x_{j}} \mathrm{~d} \Omega=-\sum_{e} \int_{\Omega^{e}} \frac{\partial\left(h_{m_{j}} v_{k}\right)}{\partial x_{j}} \bar{r}_{m_{i}} \mathrm{~d} \Omega+\int_{\Gamma} v_{k} h_{m_{j}} \bar{r}_{m_{i}} n_{j} \mathrm{~d} \Gamma \\
& \int_{\Omega} q \tau_{d_{i}} \frac{\partial \hat{\bar{r}}_{m_{i}}}{\partial x_{i}} \mathrm{~d} \Omega=-\sum_{e} \int_{\Omega^{e}} \frac{\partial\left(\tau_{d_{i}} q\right)}{\partial x_{i}} \hat{\bar{r}}_{m_{i}} \mathrm{~d} \Omega+\int_{\Gamma} q \tau_{d_{i}} \hat{\bar{r}}_{m_{i}} n_{i} \mathrm{~d} \Gamma .
\end{aligned}
$$

In above the sums extend over the element interiors $\Omega^{e}$. A proof of above equalities is given in Appendix B and also in [26].

Substituting Eqs. (23) and (24) into Eqs. (21) and (22), respectively and choosing $\hat{v}_{k}=v_{k}$ with $v_{k}=0$ on $\Gamma_{u}$ and $\hat{q}=-q$ with $q=0$ on $\Gamma_{t}$ gives

$$
\begin{aligned}
& \int_{\Omega} v_{k} \bar{r}_{m_{i}} \mathrm{~d} \Omega+\int_{\Gamma_{t}} v_{k}\left[n_{j} \bar{\sigma}_{i j}-t_{i}\right] \mathrm{d} \Gamma+\sum_{e} \int_{\Omega^{e}} \frac{1}{2} \frac{\partial\left(h_{m_{j}} v_{k}\right)}{\partial x_{j}} \bar{r}_{m_{i}} \mathrm{~d} \Omega=0, \\
& \int_{\Omega} q \bar{r}_{d} \mathrm{~d} \Omega+\sum_{e} \int_{\Omega^{e}} \frac{\partial\left(\tau_{d_{i}} q\right)}{\partial x_{i}} \hat{\bar{r}}_{m_{i}} \mathrm{~d} \Omega-\int_{\Gamma_{u_{n}}} q\left(\bar{u}_{n}-u_{n}^{p}\right) \mathrm{d} \Gamma=0 .
\end{aligned}
$$

Note that the boundary $\Gamma_{u_{t}}$ does not appear in Eq. (26) as the Dirichlet boundary for the mass balance equation coincides with $\Gamma_{u_{n}}$. Also, the last integral in Eq. (26) imposes the equality between the normal 
velocity at the boundary and its prescribed value in a weak form. This is consistent with the original stabilized equations (9) or (18) derived from balance of mass at the boundary $\Gamma_{u_{n}}$ (see Appendix A).

The integrals in the first term of Eq. (25) involving the derivatives of the pressure and the viscous stresses are treated in a distributional sense. This allows to use discontinuous pressure and stress fields across element interfaces. These integrals are computed as follows

$$
\int_{\Omega} v_{k}\left[\frac{\partial \bar{p}}{\partial x_{i}}-\frac{\partial \bar{\tau}_{i j}}{\partial x_{j}}\right] \mathrm{d} \Omega=-\sum_{e} \int_{\Omega^{e}}\left[\frac{\partial v_{k}}{\partial x_{i}} \bar{p}-\frac{\partial v_{k}}{\partial x_{j}} \bar{\tau}_{i j}\right] \mathrm{d} \Omega+\int_{\Gamma} v_{k}\left(\bar{p} \delta_{i j}-\bar{\tau}_{i j}\right) n_{j} \mathrm{~d} \Gamma .
$$

Substituting Eq. (27) into Eq. (25) noting that $\sigma_{i j}=\tau_{i j}-p \delta_{i j}$ and imposing $v_{k}=0$ on $\Gamma_{u}$ gives after simplification the stabilized integral form of the momentum equations as

$$
\begin{array}{r}
\int_{\Omega} v_{k}\left(\rho \bar{u}_{i} \frac{\partial \bar{u}_{j}}{\partial x_{j}}+\rho \bar{u}_{j} \frac{\partial \bar{u}_{i}}{\partial x_{j}}\right) \mathrm{d} \Omega+\sum_{e} \int_{\Omega^{e}}\left(-\frac{\partial v_{k}}{\partial x_{i}} \bar{p}+\frac{\partial v_{k}}{\partial x_{j}} \bar{\tau}_{i j}\right) \mathrm{d} \Omega \\
+\sum_{e} \int_{\Omega^{e}} \frac{1}{2} \frac{\partial\left(h_{m_{j}} v_{k}\right)}{\partial x_{j}} \bar{r}_{m_{i}} \mathrm{~d} \Omega-\int_{\Omega} v_{k} b_{i} \mathrm{~d} \Omega-\int_{\Gamma_{i}} v_{k} t_{i} \mathrm{~d} \Gamma=0 .
\end{array}
$$

A more convenient form of the mass balance equation is obtained integrating by parts the first integral of Eq. (26). This gives

$$
-\int_{\Omega} \frac{\partial q}{\partial x_{i}} \bar{u}_{i} \mathrm{~d} \Omega+\sum_{e} \int_{\Omega^{e}}\left[\frac{\partial\left(\tau_{d_{i}} q\right)}{\partial x_{i}}\right] \hat{\bar{r}}_{m_{i}} \mathrm{~d} \Omega+\int_{\Gamma_{u_{n}}} q u_{n}^{p} \mathrm{~d} \Gamma=0 .
$$

Note that the last integral of Eq. (28b) vanishes in rigid body and stationary free surface boundaries.

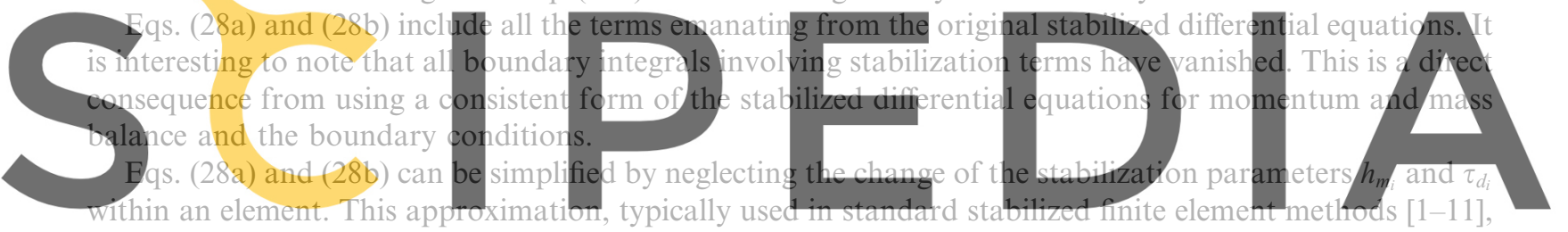

gives after rearranging some terms

Registerlfonfrees at https//www.scipedia.com to download the version without the watermark

$$
\begin{gathered}
\int_{\Omega} v_{k}\left(\rho \bar{u}_{i} \frac{\partial \bar{u}_{j}}{\partial x_{j}}+\rho \bar{u}_{j} \frac{\partial \bar{u}_{i}}{\partial x_{j}}\right)+\sum_{e} \int_{\Omega^{e}}\left(-\frac{\partial v_{k}}{\partial x_{i}} \bar{p}+\frac{\partial v_{k}}{\partial x_{j}} \bar{\tau}_{i j}\right) \mathrm{d} \Omega+\sum_{e} \int_{\Omega^{e}}\left[\frac{\partial v_{k}}{\partial x_{j}} \rho \frac{\bar{u}_{i} h_{m_{j}}}{2} \frac{\partial \bar{u}_{r}}{\partial x_{r}}\right] \mathrm{d} \Omega \\
+\sum_{e} \int_{\Omega^{e}} \frac{h_{m_{j}}}{2} \frac{\partial v_{k}}{\partial x_{j}}\left[\rho \bar{u}_{r} \frac{\partial \bar{u}_{i}}{\partial x_{r}}+\frac{\partial \bar{p}}{\partial x_{i}}-\frac{\partial \bar{\tau}_{i r}}{\partial x_{r}}-b_{i}\right] \mathrm{d} \Omega-\int_{\Omega} v_{k} b_{i} \mathrm{~d} \Omega-\int_{\Gamma_{t}} v_{k} t_{i} \mathrm{~d} \Gamma=0 .
\end{gathered}
$$

Mass balance

$$
-\int_{\Omega} \frac{\partial q}{\partial x_{i}} \bar{u}_{i} \mathrm{~d} \Omega+\sum_{e} \int_{\Omega^{e}} \tau_{d_{i}} \frac{\partial q}{\partial x_{i}} \frac{\partial \bar{p}}{\partial x_{i}} \mathrm{~d} \Omega+\sum_{e} \int_{\Omega^{e}} \tau_{d_{i}} \frac{\partial q}{\partial x_{i}} g_{i} \mathrm{~d} \Omega+\int_{\Gamma_{u_{n}}} q u_{n}^{p} \mathrm{~d} \Gamma=0
$$

where

$$
g_{i}=\rho \bar{u}_{j} \frac{\partial \bar{u}_{i}}{\partial x_{j}}-\frac{\partial \bar{\tau}_{i j}}{\partial x_{j}}-b_{i}
$$

Eqs. (28a) and (28b) or Eqs. (29a) and (29b) lead to the set of stabilized discretized equations for the velocity and pressure variables. Indeed for $v_{k}=N_{k}^{u}$ and $q=N_{j}^{p}$ the stabilized discrete Galerkin variational form is recovered.

Eqs. (29a) and (29b) incorporate terms traditionally encountered in standard stabilized formulations using the FEM. Thus, if vector $\mathbf{h}_{m}$ is assumed to be aligned with the velocity, i.e., if $h_{m_{j}}=2 \tau_{m} u_{j}$, where $\tau_{m}$ is 
an intrinsic time parameter, we find that the fourth integral in Eq. (29a) is identical to that usually introduced in Streamline Upwind Petrov Galerkin (SUPG) [3-8] and Characteristic Galerkin (CG) $[10,11]$ methods in order to remedy the instabilities due to the advection operator. The expression given by Eq. (29a) is more general and it allows to define vector $\mathbf{h}_{m}$ in a more appropiate manner to account for both streamline and transverse stabilization effects. Also by using the more general form of Eqs. (28a) and (28b) a non uniform (continuous) distribution of vector $\mathbf{h}_{m}$ can be taken into account in a consistent manner.

The third integral in Eq. (29a) has a form very similar to that found in the Galerkin Least Square (GLS) method $[9,7]$.

Note also that the divergence of the velocity term has been kept within the first integral of the momentum equation (29a). This term is usually neglected in standard SUPG and GLS approaches. In this case the presence of this term ensures consistency of the derivation. The computational relevance of this term should be verified in numerical tests.

The second and third integrals in Eq. (29b) are typically found when using GLS methods [9,7]. Note the appearance of a Laplacian of pressure term in the second integral of Eq. (29b) of the type

$$
\int_{\Omega^{e}} \tau_{d_{i}} \frac{\partial q}{\partial x_{i}} \frac{\partial \bar{p}}{\partial x_{i}} \mathrm{~d} \Omega
$$

This term has the well known property of introducing the necessary stabilization in the incompressibility equation ensuring a correct solution in the incompressible limit, while allowing the use of equal order approximations for velocity and pressure $[1,6,7,9]$.

In summary, the original stabilized differential equations for a viscous fluid are the basis for deriving, using a standard finite element Galerkin approach, a general stabilized discrete system of equations which incorporates the best features of the best known stabilized methods for simultaneously correcting both the
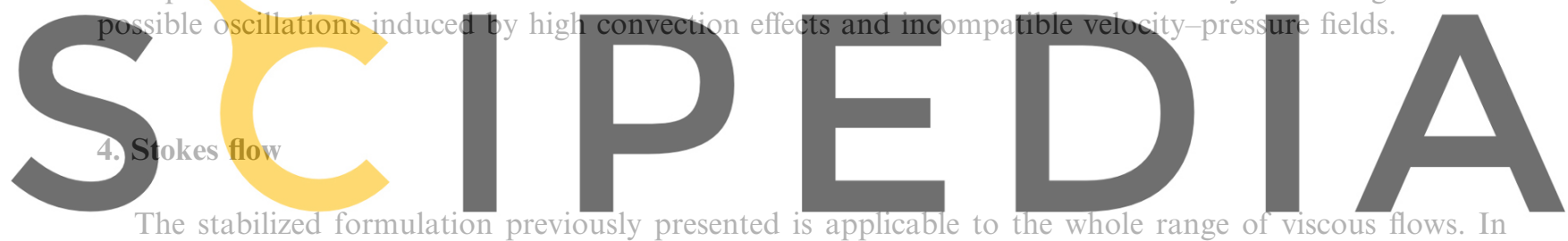

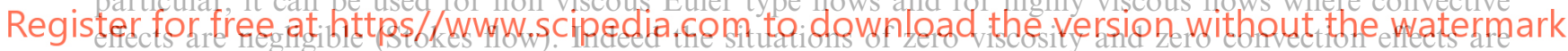

clearly non physical and they represent two limit cases of some particular fluids found in nature. It is interesting however to study the particular case of a pure viscous incompressible fiow where the effect of convection is simply neglected in the momentum equations. This assumption is typically used for modelling the deformation of metals and plastics during forming situations and also in the study of some creep problems [1,27]. The additional interest of this type of flow model is the analogy of the governing equations with those of incompressible elasticity [1,27]. Indeed, the stabilized formulation for the flow problem will be directly applicable to the analogous incompressible elastic problem.

The stabilized form of the momentum equations for a pure Stokes flow are simply obtained by neglecting the convective terms in Eqs. (1) and (3). The resulting equations can be written as

$$
\frac{\partial p}{\partial x_{i}}-\frac{\partial \tau_{i j}}{\partial x_{j}}-b_{i}-\frac{1}{2} h_{m_{j}} \frac{\partial}{\partial x_{j}}\left[\frac{\partial p}{\partial x_{i}}-\frac{\partial \tau_{i r}}{\partial x_{r}}-b_{i}\right]=0 .
$$

The next step is to express the volumetric strain rate in terms of the pressure from the momentum equations. As the convective terms are now zero the volumetric strain rate is introduced into Eq. (31) by means of the constitutive equation. Thus, substituting Eqs. (5a) and (5b) into Eq. (31) gives

$$
\frac{\partial p}{\partial x_{i}}-\frac{\partial}{\partial x_{j}}\left(2 \mu \varepsilon_{i j}\right)+\frac{\partial}{\partial x_{i}}\left(\frac{2}{3} \mu \frac{\partial u_{k}}{\partial x_{k}}\right)-b_{i}-\frac{1}{2} h_{m_{j}} \frac{\partial}{\partial x_{j}}\left[\frac{\partial p}{\partial x_{i}}-\frac{\partial}{\partial x_{r}}\left(2 \mu \varepsilon_{i r}\right)+\frac{\partial}{\partial x_{i}}\left(\frac{2}{3} \mu \frac{\partial u_{k}}{\partial x_{k}}\right)-b_{i}\right]=0 .
$$

Let us assume now the viscosity $\mu$ to be constant. Eq. (32) allows to obtain the derivatives of the volumetric strain rate as 


$$
\frac{\partial}{\partial x_{i}}\left(\frac{\partial u_{k}}{\partial x_{k}}\right)=-\frac{3}{2 \mu}\left[\frac{\partial p}{\partial x_{i}}-2 \mu \frac{\partial \varepsilon_{i j}}{\partial x_{j}}-b_{i}-\frac{1}{2} h_{m_{j}} \frac{\partial}{\partial x_{j}}\left(\frac{\partial p}{\partial x_{i}}-2 \mu \frac{\partial \varepsilon_{i r}}{\partial x_{r}}+\frac{2}{3} \mu \frac{\partial^{2} u_{r}}{\partial x_{i} \partial x_{r}}-b_{i}\right)\right]
$$

Eq. (33) can be written in a more compact form as

$$
\frac{\partial}{\partial x_{i}}\left(\frac{\partial u_{k}}{\partial x_{k}}\right)=-\frac{3}{2 \mu}\left[r_{i}-\frac{h_{m_{j}}}{2} \frac{\partial \hat{r}_{i}}{\partial x_{j}}\right]
$$

with

$$
\begin{aligned}
& r_{i}=\frac{\partial p}{\partial x_{i}}-2 \mu \frac{\partial \varepsilon_{i j}}{\partial x_{j}}-b_{i}, \\
& \hat{r}_{i}=\frac{\partial p}{\partial x_{i}}-\frac{\partial \tau_{i j}}{\partial x_{j}}-b_{i} .
\end{aligned}
$$

\section{Substituting Eq. (34) into the stabilized form of the mass balance equation given by Eq. (2) yields}

$$
\frac{\partial u_{k}}{\partial x_{k}}+\frac{3 h_{d_{i}}}{4 \mu}\left[r_{i}-\frac{h_{m j}}{2} \frac{\partial \hat{r}_{i}}{\partial x_{j}}\right]=0 .
$$

The weak form of the momentum and mass balance equations is obtained following a similar procedure for the Navier Stokes case ex
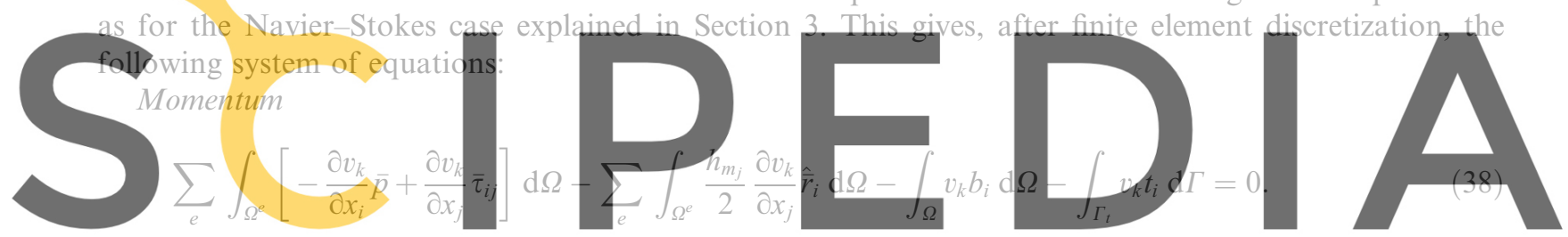

Register for free at https//www.scipedia.com to download the version without the watermark

$$
-\int_{\Omega} \frac{\partial q}{\partial x_{k}} \bar{u}_{k} \mathrm{~d} \Omega+\sum_{e} \int_{\Omega^{e}} \frac{\partial q}{\partial x_{j}}\left(\frac{3 h_{d_{i}} h_{m_{j}}}{8 \mu}\right) \hat{\bar{r}}_{i} \mathrm{~d} \Omega+\sum_{e} \int_{\Omega^{e}} q \frac{3 h_{d_{i}}}{4 \mu} \bar{r}_{i} \mathrm{~d} \Omega+\int_{\Gamma_{u_{n}}} q u_{n}^{p} \mathrm{~d} \Gamma=0,
$$

where as usual $\overline{(\cdot)}$ denotes approximate finite element values. Note that in the derivation of Eqs. (38) and (39) a uniform distribution of the stabilization parameters within each element has been assumed. In addition, the residual $\hat{r}_{i}$ has been assumed to vanish on the Dirichlet boundary.

For the sake of clarity Eq. (39) is written in the following expanded form

$$
\begin{aligned}
& -\int_{\Omega} \frac{\partial q}{\partial x_{k}} \bar{u}_{k} \mathrm{~d} \Omega+\sum_{e} \int_{\Omega^{e}} \frac{\partial q}{\partial x_{j}}\left(\frac{3 h_{d_{i}} h_{m_{j}}}{8 \mu}\right) \frac{\partial \bar{p}}{\partial x_{i}} \mathrm{~d} \Omega-\sum_{e} \int_{\Omega^{e}} \frac{\partial q}{\partial x_{j}} \frac{3 h_{d_{i}} h_{m_{j}}}{8 \mu}\left(\frac{\partial \bar{\tau}_{i k}}{\partial x_{k}}+b_{i}\right) \mathrm{d} \Omega \\
& \quad+\sum_{e} \int_{\Omega^{e}} q \frac{3 h_{d_{i}}}{4 \mu} \bar{r}_{i} \mathrm{~d} \Omega+\int_{\Gamma_{u_{n}}} q u_{n}^{p} \mathrm{~d} \Gamma=0 .
\end{aligned}
$$

Eqs. (38) and (40) provide the set of stabilized algebraic equations for computing the velocity and pressure fields after substitution of the viscous stresses and the pressure in terms of the nodal displacements and nodal pressures using Eqs. (5a) and (5b), and (19b). Indeed the pressure term in the mass balance equation allows to use equal order interpolations for velocities and pressure.

It is interesting to point out again that the stabilized discrete form provided by Eqs. (38) and (40) for the Stokes flow problem is also directly applicable to the analogous incompressible elasticity problem using equal order interpolations for displacements and pressure. 
Remark 1. The standard Laplacian of pressure form is recovered in Eq. (40) if $h_{d_{1}} h_{m_{2}}+h_{d_{2}} h_{m_{1}}=0$ (for $2 D$ problems). Otherwise, the term involving the cross derivatives of the pressure remains within the second integral of Eq. (40). The effect of this term should be validated in numerical tests.

Remark 2. Eqs. (29b) and (40) differ essentially in the method chosen to substitute the term involving the derivative of the divergence of the velocity field in the stabilized mass balance equations (see Eq. (2)). Thus, Eq. (29b) was derived making use of the convective operator and the momentum equations through the identity expressed by Eq. (11). Conversely, in the derivation of Eq. (40) use has been made of the constitutive equation to express the derivatives of the velocity divergence field in terms of rest of terms from the momentum equations (see (Eq. (33)).

\section{The transient case}

The stabilization formulation above presented is naturally extended to the transient case. The stabilized form of the momentum and mass balance equations are written now as [22,25]

Momentum

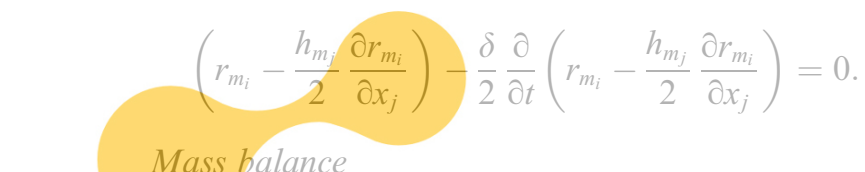

\section{Mass balance}

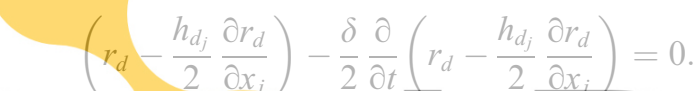

\subsection{Three steps splitting scheme}

It is interesting to derive a splitting algorithm starting with the new stabilized equations. For the sake of clarity the time stabilization terms involving $\delta$ will be neglected in Eqs. (41) and (42). Also the stabilized mass balance equation will be written in the more convenient form given by Eq. (15).

A time marching solution scheme for Eq. (41) can be written as (for $\delta=0$ )

$$
u_{i}^{n+1}=u_{i}^{n}-\frac{\Delta t}{\rho}\left[\rho \frac{\partial\left(u_{i} u_{j}\right)^{n}}{\partial x_{j}}+\frac{\partial p^{n+1}}{\partial x_{i}}-\frac{\partial \tau_{i j}^{n}}{\partial x_{j}}-b_{i}^{n}-\left(\frac{h_{m_{j}}}{2} \frac{\partial r_{m_{i}}}{\partial x_{j}}\right)^{n}\right] .
$$

The analogy of Eq. (44) with that found using the so called characteristic integration schemes [10,11] is clear if vector $\mathbf{h}_{m}$ is chosen aligned with the velocity field, i.e., $\mathbf{h}_{m}=\tau \mathbf{u}$ where $\tau$ is an intrinsic time parameter. Indeed the arbitrary form of vector $\mathbf{h}_{m}$ in Eq. (44) provides a more general procedure where the components of vector $\mathbf{h}_{m}$ can be freely chosen.

A semi-implicit time splitting or "fractional step" [10,11] algorithm can now be obtained as follows. Eq. (44) is split as

$$
u_{i}^{*}=u_{i}^{n}-\frac{\Delta t}{\rho}\left[\rho \frac{\partial\left(u_{i} u_{j}\right)}{\partial x_{j}}-\frac{\partial \tau_{i j}}{\partial x_{j}}-b_{i}-\frac{h_{m_{j}}}{2} \frac{\partial r_{m_{i}}}{\partial x_{j}}\right]^{n},
$$




$$
u_{i}^{n+1}=u_{i}^{*}-\frac{\Delta t}{\rho} \frac{\partial p^{n+1}}{\partial x_{i}} .
$$

Note that the sum of Eqs. (45) and (46) gives the original form of Eq. (44). Substituting Eq. (45) into Eq. (15) gives

$$
r_{d}^{*}-\Delta t \frac{\partial^{2} p^{n+1}}{\partial x_{i} \partial x_{i}}-\tau_{d_{i}}\left[\frac{\partial \hat{r}_{m_{i}}}{\partial x_{i}}\right]^{n+1}=0,
$$

where

$$
\begin{aligned}
& r_{d}^{*}=\frac{\partial u_{i}^{*}}{\partial x_{i}}, \\
& {\left[\frac{\partial \hat{r}_{m_{i}}}{\partial x_{i}}\right]^{n+1}=\frac{\partial}{\partial x_{i}}\left[\rho\left(\frac{\partial u_{i}}{\partial t}+u_{j} \frac{\partial u_{i}}{\partial x_{j}}\right)-\frac{\partial \tau_{i j}}{\partial x_{j}}-b_{i}\right]^{n}-\frac{\partial^{2} p^{n+1}}{\partial x_{i} \partial x_{i}} .}
\end{aligned}
$$

\section{The solution steps are the following:}

\section{Step 1: Solve explicitely for the so called "fractional" velocities $u_{i}^{*}[10,11]$ using Eq. (45).}

Step 2: Compute the pressure field $p^{n+1}$ by solving the equation for the Laplacian of pressure derived from Eq. (47). Note that this equation has the following form
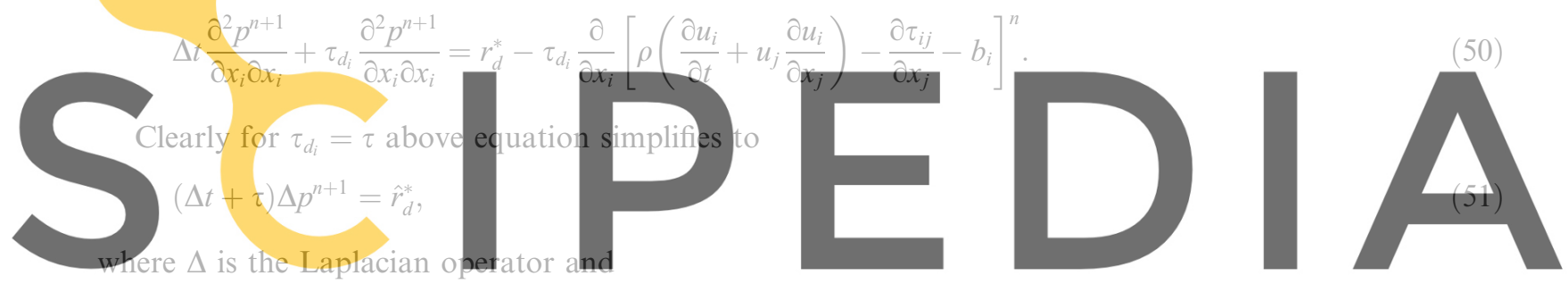

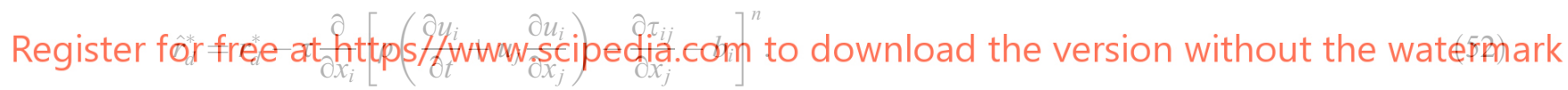

Step 3: Compute the velocities $u_{i}^{n+1}$ by using Eq. (46).

Eq. (51) differs slightly from the form typically used in fractional step schemes where the term involving $\tau$ does not appear $[10,11]$. This term, however, is essential to preserve the stability of the mixed formulation for problems where very small time increments $\Delta t$ are needed due to the stability requirements of the time integration algorithm chosen.

Obviously, other forms of above three steps transient solution scheme involving the implicit computation of $u_{i}^{n+1}$ are also possible.

Extension of these transient solution methods to the simpler Stokes problem are straightforward. The same schemes can be applied to derive enhanced algorithms for transient non linear structural dynamic problems allowing equal order interpolation for velocities and pressure as described in [28].

\section{Computation of the stabilization parameters}

Accurate evaluation of the stabilization parameters is one of the crucial issues in stabilized methods. Most of existing methods use expressions which are direct extensions of the values obtained for the simplest $1 \mathrm{D}$ case. It is also usual to accept the so called SUPG assumption, i.e., to admit that vector $\mathbf{h}_{m}$ has the direction of the velocity field. This restriction leads to instabilities when sharp layers transversal to the velocity direction are present. This additional defficiency is then corrected by adding a "shock capturing" (SC) stabilization term [17-20]. 
Let us first assume for simplicity that the stabilization parameters for the mass balance equations are the same than those for the momentum equations. This implies

$$
h_{m_{i}}=h_{d_{i}} .
$$

The problem remains now finding the value of the characteristic length vectors $h_{m_{i}}$. Indeed, the components of $\mathbf{h}_{m}$ can introduce the necessary stabilization along the streamline and transversal directions to the flow.

Excellent results have been obtained in [29] using linear triangles and tetrahedra with the following value for $h_{m_{i}}$

$$
\mathbf{h}_{m}=h_{\mathrm{s}} \frac{\mathbf{u}}{|\mathbf{u}|}+h_{\mathrm{c}} \frac{\nabla|\mathbf{u}|}{|\nabla| \mathbf{u}||},
$$

where $h_{\mathrm{s}}$ and $h_{\mathrm{c}}$ are the "streamline" and "shock capturing" contributions given by

$$
\begin{aligned}
& h_{\mathrm{s}}=\max \left(\mathbf{l}_{j}^{\mathrm{T}} \mathbf{u} /|\mathbf{u}|\right), \\
& h_{\mathrm{c}}=\max \left(\mathbf{l}_{j}^{\mathrm{T}} \frac{\boldsymbol{\nabla}|\mathbf{u}|}{|\boldsymbol{\nabla}| \mathbf{u}||}\right), \quad j=1, n_{\mathrm{s}},
\end{aligned}
$$

where $\mathbf{l}_{j}$ are the vectors defining the element sides $\left(n_{\mathrm{s}}=3\right.$ for triangles and $n_{\mathrm{s}}=6$ for tetrahedra).

An alternative method for computing $\mathbf{h}_{m}$ in a more consistent manner is explained in the next section.

\subsection{Computation of the stabilization parameters via a diminishing residual procedure}

The idea of this technique first presented in [21,22] and tested in [23-25] for advective-diffusive problems is the following. Let us assume that a finite element solution for the velocity and pressure fields has been found for a given mesh. The residual of the momentum equation corresponding to this particular solution is

$$
{ }^{1} \bar{r}_{m_{i}}=\bar{r}_{m_{i}}-\frac{1}{2} h_{m_{j}} \frac{\partial \bar{r}_{m_{i}}}{\partial x_{j}} .
$$

The average residual over an element can be defined as

$$
{ }^{1} \bar{r}_{m_{i}}^{(e)}=\frac{1}{\Omega^{(e)}} \int_{\Omega^{(e)}}^{1}{ }^{1} \bar{r}_{m_{i}} \mathrm{~d} \Omega
$$

Let us assume now that an enhanced numerical solution has been found for the same mesh and the same approximation (i.e., neither the number of elements nor the element type have been changed). This enhanced solution could be based, for instance, in a superconvergent recovery of derivatives [30,31].

The element residual for the enhanced solution is denoted ${ }^{2} \bar{r}_{m_{i}}^{(e)}$. As the element residuals must tend to zero, the following condition must be satisfied

$$
{ }^{1} \bar{r}_{m_{i}}^{(e)}-{ }^{2} \bar{r}_{m_{i}}^{(e)} \geqslant 0
$$

Above equation applies for ${ }^{1} \bar{r}_{m_{i}}^{(e)}>0$. Clearly for ${ }^{1} \bar{r}_{m_{i}}^{(e)}<0$ the inequality in Eq. (58) should be changed to $\leqslant 0$.

Eq. (58) provides a system of equations which unknowns are the characteristic length parameters. Substituting Eq. (56) into (58) and applying the identity condition in Eq. (58) gives

$$
\mathbf{h}_{m}^{(e)}=\mathbf{A}^{-1} \mathbf{f}
$$

with

$$
\begin{aligned}
A_{i j} & =2\left[\frac{{ }^{2} \partial \bar{r}_{m_{i}}^{(e)}}{\partial x_{j}}-\frac{{ }^{1} \partial \bar{r}_{m_{i}}^{(e)}}{\partial x_{j}}\right], \\
f_{i} & ={ }^{2} \bar{r}_{m_{i}}^{(e)}-{ }^{1} \bar{r}_{m_{i}}^{(e)} .
\end{aligned}
$$


The following "adaptive" algorithm can be proposed for obtaining a stabilized solution:

1. Solve for numerical values of velocities and pressure for an initial value $\mathbf{h}_{m}^{(e)}=\mathbf{h}_{o}^{(e)}$. Compute ${ }^{1} \bar{r}_{m_{i}}^{(e)}$.

2. Evaluate the enhanced velocity and pressure fields. Compute ${ }^{2} \bar{r}_{m_{i}}^{(e)}$.

3. Compute the updated value of $\mathbf{h}_{m}^{(e)}$ using Eq. (59).

4. Repeat (1)-(3) until a stable solution is found.

Above strategy can be naturally incorporated into a transient solution scheme where the value of $\mathbf{h}_{m}^{(e)}$ is updated after the solution for each time step has been found.

The assumption $\mathbf{h}_{d}=\mathbf{h}_{m}$ can be relaxed and an independent value of the characteristic length vector $\mathbf{h}_{d}$ for the mass balance equation can be found following a similar approach as described for computing $\mathbf{h}_{m}$. Further details can be found in [24,25] where this technique has been successfully tested for steady state and transient advective-diffusive problems.

\section{Concluding remarks}

The objective of the paper was to derive a stabilized formulation for finite element analysis of incompressible viscous flow problems. It has been shown that the stabilized governing equations obtained via the so called FIC procedure presented in $[21,22]$ are the basis for deriving stabilized finite element schemes for both steady state and transient situations. Moreover, the final stabilized forms obtained in all cases remedy the two main problems associated with the numerical solution of viscous flows, i.e., the lack of stability induced by high convective terms and the oscillations caused by the choice of incompatible velocitypressure fields.

The FIC method provides a natural explanation for the stabilization terms appearing in all equations, many of which have been heuristically proposed by different authors. It is interesting to note that the method extends naturally to Stokes flow problems and it also allows to derive time marching solution schemes with enhanced stabilization properties.

Extension of the FIC procedure to derive stabilized finite schemes for compressible flow problems are possible following the lines presented in $[21,22]$.

Future work remains to verify the efficiency of the "adaptive type" method proposed to compute the stabilization parameters. It is also envisaged that the FIC method could serve for deriving numerical schemes for stabilized solution of high Reynolds flows where the characteristic length parameters could naturally incorporate the stabilization properties credited to the eddy viscosity in turbulent flow models.

\section{Acknowledgements}

The author thanks Drs. R. Codina, S. Idelsohn and M. Storti and Mr. J. García and Mr. C. Sacco for many comments and suggestions.

\section{Appendix A. Balance of mass next to a boundary segment}

Let us consider the balance of mass in the triangular domain of Fig. 1 next to a boundary segment $\Gamma$. From simple observation we can write

$$
\rho\left[u_{C} h_{y}+v_{B} h_{x}\right]=\rho l u_{n}^{p},
$$

where $u_{C}$ and $v_{B}$ are the horizontal and vertical velocities at the mid points of the triangle sides and $u_{\mathrm{n}}^{p}$ is the normal velocity at the boundary. Obviously $u_{\mathrm{n}}^{p}=0$ at a solid boundary or a stationary free surface.

The velocities at points $B$ and $C$ are expressed in terms of those at point $A$ using a Taylor series approximation as

$$
\begin{aligned}
& u_{C}=u_{A}-\left.\frac{h_{x}}{2} \frac{\partial u}{\partial x}\right|_{A}, \\
& v_{B}=v_{A}-\left.\frac{h_{y}}{2} \frac{\partial v}{\partial y}\right|_{A} .
\end{aligned}
$$




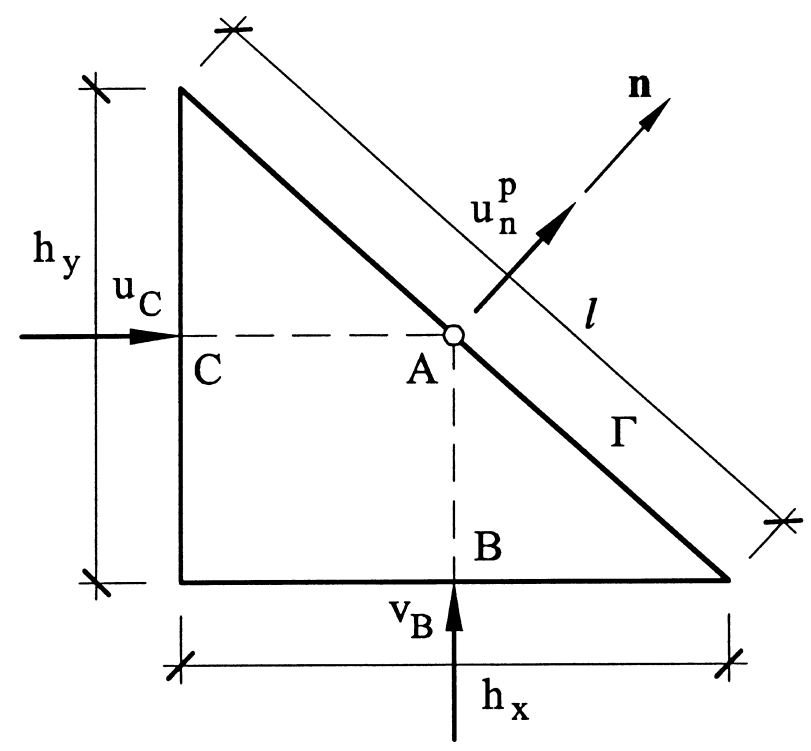

Fig. 1. Balance domain next to a boundary segment.

Substituting Eq. (A.1) into Eq. (A.2) and denoting generically $u_{A}=u$ and $v_{A}=v$ gives

$$
\left(u n_{x}+v n_{y}\right)-\frac{1}{2}\left(h_{x} n_{x}+h_{y} n_{y}\right)\left(\frac{\partial u}{\partial x}+\frac{\partial v}{\partial y}\right)=u_{\mathrm{n}}^{p} .
$$

In the derivation of Eq. (A.3) use of the identities $n_{x}=h_{y} / l$ and $n_{y}=h_{x} / l$ has been made where $n_{x}, n_{y}$ are the components of the unit normal vector $\mathbf{n}$ (see Fig. 1).

Eq. (A.3) can be further simplified to the form of Eq. (9)

$$
u_{n}-\frac{1}{2} \mathbf{h}_{d}^{\mathrm{T}} \mathbf{n} r_{d}=u_{\mathrm{n}}^{p}
$$

with

$$
\begin{aligned}
u_{n} & =u_{x} n_{x}+u_{y} n_{y}, \\
\mathbf{h}_{d} & =\left[h_{x}, h_{y}\right]^{\mathrm{T}}, \quad \mathbf{n}=\left[n_{x}, n_{y}\right]^{\mathrm{T}}, \\
r_{d} & =\frac{\partial u}{\partial x}+\frac{\partial v}{\partial y} .
\end{aligned}
$$

\section{Appendix B. Computation of integrals in a distributional sense}

The following proof is based on the ideas presented in [26]. Consider for instance the computation of

$$
\int_{\Omega} w_{j} \frac{\partial r}{\partial x_{j}} \mathrm{~d} \Omega
$$

where $w_{j}$ is a continuous function and $r$ is assumed to be a function defined at element level and which is discontinuous across the element faces. Therefore the derivatives $\partial r / \partial x_{j}$ are not defined along element sides.

Integral (B.1) can be computed in a distributional sense as

$$
\int_{\Omega} w_{j} \frac{\partial r}{\partial x_{j}} \mathrm{~d} \Omega=\sum_{e} \int_{\Omega^{e}} w_{j} \frac{\partial r}{\partial x_{j}} \mathrm{~d} \Omega+\sum_{f} \int_{\Gamma^{f}} w_{j} \llbracket r \rrbracket n_{j} \mathrm{~d} \Gamma .
$$

The first term on the righthand side represents the sum of integrals over the element interiors. The second term accounts for the jump of the discontinuous function $r$ across element faces. This term contains 
the sum of integrals computed on all faces shared by two elements. Counters $e$ and $f$ run respectively on the number of elements and faces in the mesh.

Fig. 2 illustrates the case of two triangular elements $A$ and $B$ sharing a common side $\Gamma$. For each element we consider the outward normal vector to any given side $\Gamma$. Since the jump of the discontinuous functions is computed in the normal directions, the sign of the normal makes no difference in the result of (B.2) and the jump for the case illustrated here is

$$
\llbracket r \rrbracket=(r)_{B}-(r)_{A}
$$

where $(r)_{B}$ and $(r)_{A}$ are the values of $r$ on $\Gamma$ obtained from elements $B$ and $A$, respectively. If the sign of the normal vector changes, the sign of the jump will also change so that the sign of the product $\llbracket r \rrbracket n_{j}$ will remain unchanged.

The integral of the jump along $\Gamma$ can be obtained form the contributions of each element sharing the side $\Gamma$

$$
\int_{\Gamma} w_{j} \llbracket r \rrbracket n_{j} \mathrm{~d} \Gamma=\int_{\Gamma_{B}} w_{j} r_{B} n_{j} \mathrm{~d} \Gamma-\int_{\Gamma_{A}} w_{j} r_{A} n_{j} \mathrm{~d} \Gamma
$$

Here $\Gamma_{B}$ denotes $\Gamma$ in element $A$, while $\Gamma_{A}$ represents the same face viewed from element $B$. From Fig. 2 we see that $\mathbf{n}_{A}=\mathbf{n}$ and $\mathbf{n}_{B}=-\mathbf{n}$. Therefore (B.4) can be rewritten as

$$
\int_{\Gamma} w_{j} \llbracket r \rrbracket n_{j} \mathrm{~d} \Gamma=-\int_{\Gamma_{B}} w_{j} r_{B} n_{B_{j}} \mathrm{~d} \Gamma-\int_{\Gamma_{A}} w_{j} r_{A} n_{A_{j}} \mathrm{~d} \Gamma
$$

Consequently the integral of the jump on $\Gamma$ can be decomposed into two integrals, each one involving values from only one of the two adjacent elements. The sum of such integrals for all element faces can be expressed in terms of integrals over the element boundaries as

$$
\sum_{f} \int_{\Gamma^{f}} w_{j} \llbracket r \rrbracket n_{j} \mathrm{~d} \Gamma=-\sum_{e} \int_{\Gamma^{e}} w_{j} r n_{j} \mathrm{~d} \Gamma+\int_{\Gamma} w_{j} r n_{j} \mathrm{~d} \Gamma
$$

where $\Gamma^{e}$ represents the three sides of element $e$, while $\Gamma$ represents the set of element sides lying on the boundary of $\Omega$. The second integral on the righthand side of (B.6) appears because the jump terms are computed only between two elements and not on the boundary $\Gamma$. This term will cancel out contributions from boundary edges in the first term of the righthand side.

Substituting (B.6) into (B.2) yields

$$
\int_{\Omega} w_{j} \frac{\partial r}{\partial x_{j}} \mathrm{~d} \Omega=\sum_{e}\left[\int_{\Omega^{e}} w_{j} \frac{\partial r}{\partial x_{j}} \mathrm{~d} \Omega-\int_{\Gamma^{e}} w_{j} r n_{j} \mathrm{~d} \Gamma\right]+\int_{\Gamma} w_{j} r n_{j} \mathrm{~d} \Gamma .
$$

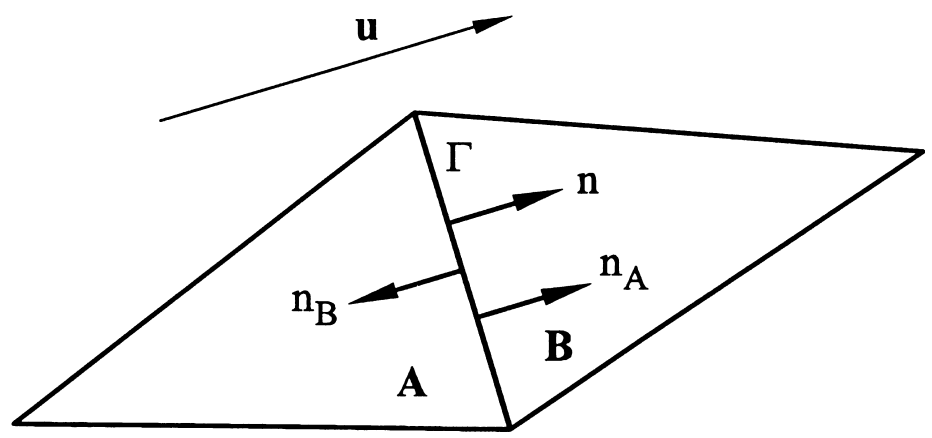

Fig. 2. Interface for computing the jump between two elements. 
The last step consists in integrating by parts the integrals over the element interiors, i.e.,

$$
\int_{\Omega^{e}} w_{j} \frac{\partial r}{\partial x_{j}} \mathrm{~d} \Omega=-\int_{\Omega^{e}} \frac{\partial w_{j}}{\partial x_{j}} r \mathrm{~d} \Omega+\int_{\Gamma^{e}} w_{j} r n_{j} \mathrm{~d} \Gamma .
$$

Substituting (B.8) into (B.7) gives finally

$$
\int_{\Omega} w_{j} \frac{\partial r}{\partial x_{j}} \mathrm{~d} \Omega=-\sum_{e} \int_{\Omega^{e}} \frac{\partial w_{j}}{\partial x_{j}} r \mathrm{~d} \Omega+\int_{\Gamma} w_{j} r n_{j} \mathrm{~d} \Gamma .
$$

This coincides with Eqs. (23) and (24) for $w_{j}=v_{k} h_{m_{j}}$ and $r=\bar{r}_{m_{i}}$ in Eq. (23) and $w_{j}=q \tau_{d_{j}}$ and $r=\hat{\bar{r}}_{m_{i}}$ in Eq. (24).

\section{References}

[1] O.C. Zienkiewicz, R.L. Taylor, The Finite Element Method, McGraw-Hill, vol. 1, 1989; vol. 2, 1991.

[2] R. Codina, A finite element model for incompressible flow problems, Ph.D. Thesis, Universitat Politécnica de Catalunya, Barcelona, June 1992.

[3] A. Brooks, T.J.R. Hughes, Streamline upwind/Petrov-Galerkin formulation for convection dominated flows with particular emphasis on the incompressible Navier-Stokes equations, Comput. Methods Appl. Mech. Engrg. 32 (1982) 199-259.

[4] T.J.R. Hughes, M. Mallet, A new finite element formulations for computational fluid dynamics: III. The generalized streamline operator for multidimensional advective-diffusive systems, Comp. Methods Appl. Mech. Engrg. 58 (1986) $305-328$.

[5] P. Hansbo, A. Szepessy, A velocity-pressure streamline diffusion finite element method for the incompressible Navier-Stokes equations, Comp. Methods Appl. Mech. Engrg. 84 (1990) 175-192.

[6] T.J.R. Hughes, L.P. Franca, M. Balestra, A new finite element formulation for computational fluid dynamics. V Circumventing the Babuska-Brezzi condition: A stable Petrov-Galerkin formulation of the Stokes problem accomodating equal order interpolations, Comp. Methods Appl. Mech. Engrg. 59 (1986) 85-89.

[7] L.P. Franca, S.L. Frey, Stabilized finite element methods: II. The incompressible Navier-Stokes equations, Comput. Methods Appl. Mech. Engrg. 99 (1992) 209-233.

[8] T.J.R. Hughes, G. Hauke, K. Jansen, Stabilized finite element methods in fluids: Inspirations, origins, status and recent developments, in: T.J.R. Hughes, E. Oñate, O.C. Zienkiewicz (Eds.), Recent Developments in Finite Element Analysis (A Book Dedicated to Robert L. Taylor) International Center for Numerical Methods in Engineering, Barcelona, Spain, 1994, pp. $272-292$.

[9] T.J.R. Hughes, L.P. Franca, G.M. Hulbert, A new finite element formulation for computational fluid dynamics: VIII. The Galerkin/least-squares method for advective-diffusive equations, Comput. Methods Appl. Mech. Engrg. 73 (1989) $173-189$.

[10] O.C. Zienkiewicz, R. Codina, A general algorithm for compressible and incompressible flow. Part I: The split characteristic based scheme, Int. J. Num. Meth. Fluids 20 (1995) 869-885.

[11] O.C. Zienkiewicz, K. Morgan, B.V.K. Satya Sai, R. Codina, M. Vázquez, A general algorithm for compressible and incompressible flow. Part II: Tests on the explicit form, Int. J. Num. Meth. Fluids 20 (8-9) (1995) 886-913.

[12] T.J.R. Hughes, Multiscale phenomena: Greens functions subgrid scale models, bubbles and the origins of stabilized methods, Comput. Methods Appl. Mech. Engrg. 127 (1995) 387-401.

[13] R. Codina, A stabilized finite element method for generalized stationary incompressible flows, Publication PI-148, CIMNE, Barcelona, February 1999.

[14] F. Brezzi, M.O. Bristeau, L.P. Franca, M. Mallet, G. Rogé, A relationship between stabilized finite element methods and the Galerkin method with bubble functions, Comput. Methods Appl. Mech. Engrg. 96 (1992) 117-129.

[15] F. Brezzi, D. Marini, A. Russo, Pseudo residual-free bubbles and stabilized methods, in: J. Periaux et al. (Eds.), Computational Methods in Applied Sciences, Wiley, Chichester, 1996.

[16] F. Brezzi, L.P. Franca, T.J.R. Hughes, A. Russo, “ $b=\int g$ ”, Comput. Methods Appl. Mech. Engrg. 145 (1997) $329-339$.

[17] A. Mizakami, T.J.R. Hughes, A Petrov-Galerkin finite element method for convection dominated flows: an accurate upwinding technique for satisfying the maximum principle, Comput. Methods Appl. Mech. Engrg. 50 (1985) 181-193.

[18] T.J.R. Hughes, M. Mallet, A new finite element formulations for computational fluid dynamics: IV. A discontinuity capturing operator for multidimensional advective-diffusive system, Comput. Methods Appl. Mech. Engrg. 58 (1986) 329-336.

[19] A.C. Galea problems, Comput. Methods Appl. Mech. Engrg. 68 (1988) 83-95.

[20] R. Codina, A discontinuity-capturing crosswind dissipation for the finite element solution of the convection-diffusion equation, Comput. Methods Appl. Mech. Engrg. 110 (1993) 325-342.

[21] E. Oñate, On the stabilization of numerical solution for advective-diffusive transport and fluid flow problems, Publication PI-81, CIMNE, Barcelona, October 1996.

[22] E. Oñate, Derivation of stabilized equations for advective-diffusive transport and fluid flow problems, Comput. Methods Appl. Mech. Engrg. 151 (1/2) (1998) 233-267.

[23] E. Oñate, J. García, S. Idelsohn, Computation of the stabilization parameter for the finite element solution of advective-diffusive problems, Int. J. Num. Meth. Fluids 25 (1997) 1385-1407. 
[24] E. Oñate, J. García, S. Idelsohn, An alpha-adaptive approach for stabilized finite element solution of advective-diffusive problems with sharp gradients, in: P. Ladeveze, J.T. Oden (Eds.), New Adv. Adaptive Comp. Met. Mech., Elsevier, New York, 1998.

[25] E. Oñate, M. Manzan, A general procedure for deriving stabilized space-time finite element methods for advective-diffusive problems, Publication PI-133, CIMNE, Barcelona, July 1998.

[26] F. Ilinca, J.F. Hétu, D. Peletier, On stabilized finite element formulations for incompressible advective-diffusive transport and fluid flow problems, AIAA-97-1863, Presented at AIAA CFD Conference, Snowman, USA, July 1997; Comp. Methods Appl. Mech. Engrg., in press.

[27] O.C. Zienkiewicz, P.C. Jain, E. Oñate, Flow of solids during forming and extrussion: some aspects of numerical solution, Int. J. Solids Struc. 14 (1978) 15-38.

[28] O.C. Zienkiewicz, J. Rojek, R.L. Taylor, M. Pastor, Triangles and tetrahedra in explicit dynamic codes for solids, Int. J. Num. Methods Engrg. 43 (1998) 565-583.

[29] E. Oñate, J. García, A stabilized finite element method for incompressible flows involving free surface waves, Publication PI-160, CIMNE, Barcelona, 1999.

[30] O.C. Zienkiewicz, J.Z. Zhu, The supercorvergent patch recovery (SPR) and adaptive finite element refinement, Comp. Methods Appl. Mech. Engrg. 101 (1992) 207-224.

[31] N.E. Wiberg, F. Abdulwahab, X.D. Li, Error estimation and adaptive procedures based on superconvergent patch recovery, Archives Comput. Meth. Engrg. 4 (3) (1997) 203-242. 